\title{
In situ FTIR analysis in determining possible chemical reactions for peroxide crosslinked LDPE in the presence of triallyl cyanurate
}

\begin{abstract}
Most of the current methods used in the investigation of crosslinking reactions between low density polyethylene (LDPE) and dicumylperoxide (DCP) alone or with DCP/triallyl cyanurate (TAC) systems are normally inconclusive in determining its detailed reaction scheme. In this paper, an attempt to determine the reactions mechanism of this particular system was investigated using electron spin resonance (ESR), nuclear magnetic resonance (NMR) and Fourier transform infrared (FTIR). It was found that the ESR was unsuccessful in gaining insight into reaction mechanisms. Similarly, at the low degree of crosslinking necessary to allow samples to swell, the NMR technique was not suitable to illustrate structural changes after crosslinking. On the other hand, in situ FTIR was proven to be the most elucidating technique, positively indicating oxidation, full peroxide dissociation and indications that TAC reacted by forming both allyl and alkyl radicals. These radicals then appeared to take part in additional reaction incorporating into the network (improving the crosslinking efficiency) or possibly by self-polymerisation either as an independent species or more probably within the network
\end{abstract}

